TRANSACTIONS OF THE

AMERICAN MATHEMATICAL SOCIETY

Volume 186, December 1973

\title{
WEIGHTED GROTHENDIECK SUBSPACES
}

\author{
BY \\ JOÃO B. PROLLA AND SILVIO MACHADO
}

\begin{abstract}
Let $V$ be a family of nonnegative upper semicontinuous functions on a completely regular Hausdorff space $X$. For a locally conver Hausdorff space $E$, let $C V_{\infty}(X ; E)$ be the corresponding Nachbin space, that is, the rector space of all continuous functions $f$ from $X$ into $E$ such that $v f$ vanishes at infinity for all $v \in V$, endowed with the topology given by the seminorms of the type $f 1 \rightarrow$ sup $\{v(x) \bar{p}(\bar{f}(x)) ; x \in X\}$, where $v \in V$ and $p$ is a continuous seminom on $E$. Given a vector subspace $L$ of $C V_{\infty}(X ; E)$, the set of all pairs $x, y \in X$ such that either $0=\delta_{x}\left|L=\delta_{y}\right| L$ or there is $t \in R_{,} t \neq 0$, such that $0 \neq \delta_{x}\left|L=t \delta_{y}\right| L$, is an equivalence relation, denoted by $G_{L}$, and we define for $(x, y) \in G_{L}^{y}, g(x, y)=0$ or $t$, accordingly. The subsets $K S_{L}$, resp. $W S_{L}$, where $g(x, y) \geq 0$, resp. $g(x, y) \in\{0,1\}$ are likewise equivalence relations. The $G$-hull (resp. $K S$-hull, WS-hull) of $L$ is the rector subspace $\left\{f \in C V_{\infty}(X ; E)\right.$; $f(x)=g(x, y) f(y)$ for all $(x, y) \in G_{L}$ (resp. $\left.\left.K S_{L}, W S_{L}\right)\right\}$ and $L$ is a $G$-space (resp. $K S$-space, WS-space) if its $G$-hull (resp. $K S$-hull, WS-hull) is contained in its closure. This paper is devoted to the characterization, by invariance properties, of the G-spaces resp. $K S$-spaces and WS-spaces of a given Nachbin space $C V_{\infty}(X ; E)$. As an application we derive an infinite-dimensional Weierstrass polynomial approximation theorem, and a Tietze extension theorem for Banach space valued compact mappings.
\end{abstract}

1. Introduction. Let $X$ be a completely, regular Hausdorff space, and let $E$ be a locally convex Hausdorff space, not reduced to $\{0\}$. The vector space of all $E$-valued continuous functions on $X$ will be denoted by $C(X ; E)$, while $C_{b}(X ; E)$ denotes the subspace of all $f \in C(X ; E)$ which are bounded. A weight on $X$ is a nonnegative upper semicontinuous function on $X$. A set $V$ of weights on $X$ is directed if, for all $u, v \in V$, there are $w \in V$ and $t>0$ such that $u \leq t w$ and $v \leq t w$ pointwise. All sets of weights considered will be directed and $V>0$, i.e., given $x \in X$ there is $v \in V$ such that $v(x)>0$. Given such a $V$ the Nacbbin space $C V_{\infty}(X ; E)$ is the vector subspace of all $f \in C(X ; E)$ such that vf vanishes at infinity, for every $v \in V$, endowed with the topology determined by the seminorms $f \mapsto \sup \{v(x) p(f(x)) ; x \in X\}$, where $v \in V$, and $p \in s(E)$, a fixed

Received by the editors April 7, 1973.

AMS (MOS) subject classifications (1970). Primary 46105, 46E10; Secondary 46E40, $46 \mathrm{M} 05$.

Key words and phrases. Nachbin spaces of continuous vector-valued functions, Grothendieck spaces, Kakutani-Stone spaces, Weierstrass-Stone spaces, polynomial algebras, latticial subspaces, Lindenstrauss-Wulbert subspaces, compact mappings. 
determining set of continuous seminorms on $E$. The following definitions are from Blatter [2].

Let $L$ be a vector subspace of $C V_{\infty}(X ; E)$. The set $G_{L}$ of all pairs $x, y \in X$ such that either $0=\delta_{x}\left|L=\delta_{y}\right| L$ or there exists $t \in R, t \neq 0$, such that $0 \neq$ $\delta_{x}\left|L=t \delta_{y}\right| L$, is an equivalence relation for $X$, and we define a map $g: G_{L^{-}} \rightarrow \mathbf{R}$ as follows:

$$
\begin{aligned}
& g(x, y)=0, \quad \text { if } 0=\delta_{x}\left|L=\delta_{y}\right| L ; \\
& g(x, y)=t, \quad \text { if } 0 \neq \delta_{x}\left|L=t \delta_{y}\right| L .
\end{aligned}
$$

The sets $K S_{L}$ and $W S_{L}$ of all pairs $(x, y) \in G_{L}$ such that $g(x, y) \geq 0$ and $g(x, y) \in\{0,1\}$, respectively, are likewise equivalence relations for $X$. Let $\Delta \in\{G, K S, W S\}$. The vector subspace $\Delta(L)=\left\{f \in C V_{\infty}(X ; E) ; f(x)=g(x, y) f(y)\right.$ for atl $\left.(x, y) \in \Delta_{L}\right\}$ is called the $\Delta$-bull of $L$ in $C V_{\infty}(X ; E)$, and $L$ is called a $\Delta$-space is $\Delta(L)$ is the closure of $L$ in $C V_{\infty}(X ; E)$. Notice that, by the hypothesis made on $V$, the closure of $L$ is always contained in $\Delta(L)$. The letters $G, K S$, and WS stand for Grothendieck, Kakutani-Stone, and Weierstrass-Stone respectively.

The object of the present paper is to characterize, by invariance properties, those subspaces $L$ which are $\Delta$-spaces. Our results are as follows. Let $A(\Delta)$ be the algebra of all $b \in C_{b}(X ; R)$ which are constant on the equivalence classes modulo $\Delta_{L}$. Assume that $A(\Delta)$ separates the equivalence classes modulo $\Delta_{L}$. (This is automatically satisfied in the case $\Delta=W S$.) Let $M=\left\{u^{*}(f) ; u^{*} \in E^{*}, f \in L\right\}$. Then:

(1) $L$ is a WS-space, if $L$ is a polynomial algebra such that $M$ is localizable under itself in $C V_{\infty}(X ; \mathrm{K})$.

(2) $L$ is a $K S$-space, if $L$ is a latticial subspace.

(3) $L$ is a $G$-space, if $L$ is a Lindenstrauss-Wulbert subspace (see Definition 3.10).

In the spirit of Stone's paper [11], we apply some of these results to reobtain vector-valued versions of some classical theorems.

The following elementary result will be used many times.

1.1 Lemma. Let $L$ be a vector subspace of $C V_{\infty}(X ; E)$. The following statements are equivalent.

(1) $M=\left\{u^{*}(f) ; u^{*} \in E^{*}, f \in L\right\}$ is a vector subspace of $C V_{\infty}(X ; K)$ and $M \otimes E \subset L$. If $L$ is closed, $M$ is closed, too.

(2) Given $u^{*} \in E^{*}, f \in L$, and $u \in E$, then $u^{*}(f) u \in L$.

Proof. It is obvious that (1) implies (2). Conversely, assume (2). $M$ is clearly invariant under scalar multiplication. Let $u^{*}, v^{*} \in E^{*}$, and $f, b \in L$ be given. We may assume that $u^{*}$ and $v^{*}$ are linearly independent. Choose $u, v \in E$ such that $u^{*}(u)=v^{*}(v)=1, u^{*}(v)=v^{*}(u)=0$. By (2), $k=u^{*}(f) u+v^{*}(b) v$ belongs 
to L. Let $w^{*}=u^{*}+v^{*}$. Then $u^{*}(f)+v^{*}(b)=w^{*}(k) \in M$. Obviously $M \otimes E C L$. Suppose now that $L$ is closed. Let $f$ be in the closure of $M$. Choose $u^{*} \in E^{*}$ and $u \in E$ such that $u^{*}(u)=1$. Set $b=f \otimes u$. Given $v \in V, p \in s(E)$, and $\epsilon>0$, there exists $w^{*}(k) \in M$ such that $v(x)\left|f(x)-w^{*}(k(x))\right|<\epsilon /(1+p(u))$ for all $x \in X$. Then $w^{*}(k) u \in L$ and $v(x) p\left(b(x)-w^{*}(k(x)) u\right)<\epsilon$ for all $x \in X$. Since $L$ is closed, $b \in L$. Therefore, $f=u^{*}(b) \in M$, and $M$ is closed.

\section{Weierstrass-Stone spaces.}

2.1 Definition. Let $E$ and $F$ be two nonzero topological vector spaces. For each integer $n \geq 1, P_{f}\left({ }^{n} E ; F\right)$ denotes the vector subspace of $C(E ; F)$ generated by the set of all maps of the form $x \mapsto u^{*}(x)^{n} u$, where $u^{*} \in E^{*}$, the topological dual of $E$, and $u \in F$. The elements of $P,\left({ }^{n} E ; F\right)$ are called $n$-bomogeneous continuous polynomials of finite type from $E$ into $F$. The vector subspace generated by the union of all $P_{f}\left({ }^{n} E ; F\right), n \geq 1$, and the constant maps, is denoted by $P_{f}(E ; F)$. Similarly, $L_{f}\left({ }^{n} E ; F\right)$, for each integer $n \geq 1$, denotes the vector space generated by the set of all maps of the form $\left(x_{1}, \cdots, x_{n}\right) \mapsto u_{1}^{*}\left(x_{1}\right) \cdots u_{n}^{*}\left(x_{n}\right) u$ where $u_{1}^{*}, \cdots, u_{n}^{*} \in E^{*}$ and $u \in F$. The elements of $L_{f}\left({ }^{n} E ; F\right)$ are called $n$-linear continuous mappings of finite type from $E^{n}$ into $F$.

2.2 Lemma. Let $L$ be a vector subspace of $C V_{\infty}(X ; E)$. The following statements are equivalent.

(1) Given $b_{1}, \cdots, b_{n} \in L$ and $T \in L_{f}\left({ }^{n} E ; E\right)$, where $n \geq 1$, then $T\left(b_{1}, \cdots, b_{n}\right) \in L$.

(2) Given $b \in L$ and $p \in P\left({ }^{n} E ; E\right)$, where $n \geq 1$, then $p(b) \in L$.

(3) $M=\left\{u^{*}(b) ; u^{*} \in E^{*}, b \in L\right\}$ is an algebra contained in $C V V_{\infty}(X ; K)$ sucb tbat $M \otimes E \subset L$.

(4) (a) Given $b \in L, u^{*} \in E^{*}$, and $u \in E$, then $u^{*}(b) u \in L$.

(b) There exists a continuous map $T: E \times E \rightarrow E$ sucb that $T(v, v) \neq 0$ for some $v \in E$ for which $T(a v, b v)=a b T(v, v)$ for all $a, b \in \mathbf{K}$ and $T(b, k) \in L$ for all $b, k \in L$.

Proof. It is obvious that (1) implies (2). Assume (2). By the Lemma 1.1 the set $M$ is a vector subspace of $C V_{\infty}(X ; K)$ such that $M \otimes E C L$. Since $4 u^{*}(b) v^{*}(k)=\left[\dot{u}^{*}(b)+v^{*}(k)\right]^{2}-\left[u^{*}(b)-v^{*}(k)\right]^{2}$, all that remains to prove is that $u^{*}(b)^{2}$ belongs to $M$ for any $u^{*} \in E^{*}$ and $b \in L$. If $u^{*}=0$, there is nothing to prove. Let $u \in E$ be such that $u^{*}(u)=1$, and define $p(t)=u^{*}(t)^{2} u$ for all $t \in E$. By (2), $k=p(b) \in L$. Since $u^{*}(k)=u^{*}(b)^{2}$, the set $M$ is an algebra. Assume now (3). Part (a) follows immediately. To prove part (b) of (4), consider $u^{*} \in E^{*}$ and $v \in E$ with $u^{*}(v)=1$. Define $T(s, t)=u^{*}(s) u^{*}(t) v$ for all $s, t \in E$. Then $T$ satisfies all requirements. Finally, assume (4). Let $b_{1}, \cdots, b_{n} \in L$, $u_{1}^{*}, \cdots, u_{n}^{*} \in E^{*}$, and $u \in E$ be given. We claim that $u_{1}^{*}\left(b_{1}\right) \cdots u_{n}^{*}\left(b_{n}\right) u \in L$. 
The proof is by induction. If $n=1$, the conclusion follows from part (a). Assume that the conclusion is true for $m$. Then, given $b_{i}, u_{i}^{*}, i=1, \cdots, m+1$, we have $u_{1}^{*}\left(b_{1}\right) \cdots u_{m}^{*}\left(b_{m}\right) v \in L$. Call it $b$. Let $k=u_{m+1}^{*}\left(b_{m+1}\right) v$. Then $k \in L$, by part (a), and $T(b, k) \in L$, by part (b). Choose $v^{*} \in E^{*}$ such that $v^{*}(T(v, v))=1$. By part (a), $v^{*}(T(b, k)) u=u_{1}^{*}\left(b_{1}\right) \cdots u_{m+1}^{*}\left(b_{m+1}\right) u \in L$, and the proof is complete.

2.3 Definition. A vector subspace $L \subset C V_{\infty}(X ; E)$ is called a polynomial algebra if any of the equivalent statements (1)-(4) is true.

2.4 Remark. The name "polynomial algebra" was introduced by Wulbert (unpublished) for vector subspaces satisfying (2), when one allows all continuous polynomials and not just those of finite type. Subspaces $L$ of $C(X ; E)$, for $X$ compact and $E$ a real Banach space, satisfying (1) were considered by Pel czyński [9], but he allowed $T$ to be any multilinear continuous mapping and $L$ contained the constants. Blatter, in [2], considered subspaces of $C_{\infty}(X ; E)$, for $X$ locally compact and $E$ a real Banach space, satisfying (4).

2.5 Remark. When $E=K, L \subset C V_{\infty}(X ; K)$ satisfies (1)-(4) if, and only if, $L$ is an algebra under pointwise operations, and the equivalence relation $W S_{L}$ is just the usual equivalence relation $X / L$ defined by $x \sim y$ if, and only if, $b(x)=$ $b(y)$ for all $b \in L$. We recall that, in this case, $L$ is called localizable under itself in $C V_{\infty}(X ; K)$ (see Nachbin [7]) when the following condition holds true: A function $f \in C V_{\infty}(X ; K)$ is in the closure of $L$ if (and always if), for any $v \in V$, any $\epsilon>0$, and any equivalence class $Y \subset X$ modulo $X / L$, there exists $b \in L$ such that $v(x)|f(x)-b(x)|<\epsilon$ for all $x \in Y$. It is immediate that $L$ is localizable if, and only if, $L$ is a WS-space. Theorem 4 of Nachbin [7] gives a sufficient condition for $L$ to be localizable in $C V_{\infty}(X ; \mathbf{K})$.

2.6 Proposition. Suppose that $C V_{\infty}(X ; E)$ is a polynomial algebra and let $L$ be a vector subspace of $C V_{\infty}(X ; E)$. The WS-bull of $L$ is a polynomial algebra such that $M=\left\{u^{*}(b) ; u^{*} \in E^{*}, b \in W S(L)\right\}$ is localizable in $C V_{\infty}(X ; K)$.

Proof. Let $b \in W S(L), u^{*} \in E^{*}, u \in E$, and $n \geq 1$ be given. Let $k=u^{*}(b)^{n} u$. Since $C V_{\infty}(X ; E)$ is a polynomial algebra, $k \in C V_{\infty}(X ; E)$. Let $(x, y) \epsilon$ $W S_{L}$. Then $k(x)=u^{*}(b(x))^{n} u=u^{*}(g(x, y) b(y))^{n} u=g(x, y) k(y)$, since $g(x, y) \in\{0,1\}$. Hence $k \in W S(L)$, and therefore $W S(L)$ is a polynomial algebra. On the other hand, let $f \in C V_{\infty}(X ; K)$ belong to WS(M). Choose $u \in E$ and $u^{*} \in E^{*}$ such that $u^{*}(u)=1$. Then $b=f \otimes u$ belongs to $W S(L)$, because the formation of WS -hulls is idempotent. Therefore, $u^{*}(b)=f \in M$, and $M$ is localizable in $C V_{\infty}(X ; \mathrm{K})$.

2.7 Corollary. Suppose that $C V_{\infty}(X ; E)$ is a polynomial algebra and let $L$ be a Weierstrass.Stone space contained in $C V_{\infty}(X ; E)$. Then its closure is a poly. 
nomial algebra and $M=\left\{u^{*}(b) ; u^{*} \in E^{*}, b \in L\right\}$ is localizable in $C V_{\infty}(X ; K)$.

2.8 Lemma. Let $A$ be a selfadjoint subalgebra of $C(X ; K)$ sucb that every $b \in A$ is bounded on the support of every $v \in V$, and let $L$ be a vector subspace of $C V_{\infty}(X ; E)$ which is an A-module. Then, $f \in C V_{\infty}(X ; E)$ is in the closure of $L$ if (and always only if), for every equivalence class $Y \subset X$ modulo $X / A$, every $v \in V$, every $p \in s(E)$, and every $\epsilon>0$, there is $k \in L$ sucb that for all $x \in Y$, $v(x) p(f(x)-k(x))<\epsilon$.

Proof. This is an immediate corollary to Theorem 2 of [8].

2.9 Theorem. Let $L$ be a vector subspace of $C V_{\infty}(X ; E)$ which is a polynomial algebra sucb that $M=\left\{u^{*}(b) ; u^{*} \in E^{*}, b \in L\right\}$ is localizable in $C V_{\infty}(X ; K)$. Then $L$ is a WS.space.

Proof. Let $A=A(W S)$ be the algebra of all $f \in C_{b}(X ; \mathbf{R})$ which are constant on the equivalence classes modulo $W S_{L}$. Notice that $W S(M)$ is an $A$-module. Therefore, since $M$ is localizable; $\bar{M}$ is an $A$-module. Let $b \in W S(L)$. Let $Y \subset X$ be an equivalence class modulo $X / A$, let $\nu \in V, p \in s(E)$, and $\epsilon>0$ be given. Since $M$ and $A$ define the same equivalence relation on $X$, namely $W S_{L}$, $b$ is constant on $Y$. Let $u \in E$ be this constant value. If $u=0$, then $0 \in M \otimes E$ is such that $v(x) p(b(x)-0)=0<\epsilon$ for all $x \in Y$. If $u \neq 0$, there exists $f \in L$ whose constant value on $Y$, say $t$, is not zero. Let $u^{*} \in E^{*}$ be such that $u^{*}(t)=1$. Then $k=u^{*}(f) u$ belongs to $M \otimes E$ and $v(x) p(b(x)-k(x))=0<\epsilon$ for all $x \in Y$. By Lemma $2.8, b$ belongs to the closure of $\bar{M} \otimes E$. Since $\bar{M} \otimes E$ is contained in the closure of $L, L$ is a Weierstrass-Stone space.

2.10 Remark. A subset $L$ of $C V_{\infty}(X ; E)$ is called separating if for any $x, y \in X$ with $x \neq y$, there is $b \in L$ such that $b(x) \neq b(y)$, i.e., if $W S_{L}$ reduces to the diagonal. In this case, WS $(L)$ is precisely the set of all $b \in C V_{\infty}(X ; E)$ which vanish on the subset $Z(L)=\{x \in X ; f(x)=0$ for all $f \in L\}$. When $Z(L)=\varnothing$, we say that $L$ is everywhere different from zero.

2.11 Corollary. Let $L$ be as in Theorem 2.9. Suppose that $L$ is separating. Then, $f \in C V_{\infty}(X ; E)$ belongs to the closure of $L$ if, and only if, $f$ vanishes on the set $Z(L)$.

2.12 Corollary. Let $L$ be as in Theorem 2.9. Suppose that $L$ is separating and everywbere different from zero. Then $L$ is dense in $C V V_{\infty}(X ; E)$.

3. Kakutani-Stone and Grothendieck spaces. In this paragraph $E$ is a real locally convex Hausdorff space.

3.1 Definition. A subset $M \subset C(X ; \mathbf{R})$ is a lattice if $\sup (f, b) \in M$ and inf $(f, b) \in M$ whenever $f, b \in M$. 
3.2 Theorem (Nachbin [7]). Let $M$ be a sublattice of $C V_{\infty}(X ; \mathbf{R})$. A function $f \in C V_{\infty}(X ; \mathbf{R})$ is in the closure of $M$ if, and only if, for every pair $x, y \in X$ and every $\epsilon>0$, there exists $b \in M$ sucb that $|b(x)-f(x)|<\epsilon$ and $|b(y)-f(y)|<\epsilon$.

3.3 Remark. It follows from Definition 3.1 that the $K S$-hull of any vector subspace $M$ of $C V V_{\infty}(X ; \mathbf{R})$ is a vector lattice.

3.4 Theorem. Any vector sublattice of $C V_{\infty}(X ; \mathbf{R})$ is a Kakutani-Stone space.

Proof. Let $M$ be a vector sublattice of $C V_{\infty}(X ; \mathbf{R})$ and let $b \in K S(M)$. Let $x, y \in X$ be given arbitrarily.

Case I. $0=\delta_{x} \mid M$ or $0=\delta_{y} \mid M$. Suppose $0=\delta_{x} \mid M$. Then $g(x, x)=0$ and $b(x)=0$. If $(x, y) \in K S_{M}$, then $b(y)=0$ too, and $0 \in M$ agrees with $b$ at $x$ and $y$. If $(x, y) \notin K S_{M}$, then there is some $f \in M$ such that $f(x)=0=b(x)$ and $f(y)=b(y)$. The case $0=\delta_{y} \mid M$ is analogous.

Case II. $0 \neq \delta_{x} \mid M$ and $0 \neq \delta_{y} \mid M$. If $(x, y) \in K S_{M}$, then $0 \neq \delta_{x}\left|M=r \delta_{y}\right| M$ for some $r>0$. Choose $f \in M$ such that $f(x)=b(x)$. Then $f(y)=b(y)$, because both $f$ and $b$ belong to $K S(M)$. If $(x, y) \notin K S_{M}, \delta_{x}\left|M \neq r \delta_{y}\right| M$ for all $r>0$. Choose $f_{x} \in M$ such that $f_{x}(x)=1$. If $f_{x}(y) \leq 0$, we can in fact suppose $f_{x}(y)=0$, by taking $\sup \left(f_{x}, 0\right) \in M$, if necessary. Choose $f_{y} \in M$ with $f_{y}(y)=1$. Then, the function $f=\left(b(x)-b(y) f_{y}(x)\right) f_{x}+b(y) f_{y}$ belongs to $M$ and is such that $f(x)=b(x)$ and $f(y)=b(y)$. If $f_{x}(y)>0$, there is $b_{y} \in M$ such that $b_{y}(x) \neq\left(f_{x}(y)\right)^{-1} b_{y}(y)$. Hence the system

$$
a f_{x}(x)+b b_{y}(x)=b(x), \quad a f_{x}(y)+b b_{y}(y)=b(y)
$$

can be solved for $a$ and $b$. Then $f=a f_{x}+b b_{y} \in M$ agrees with $b$ at $x$ and $y$. From Theorem 3.2, $b$ belongs to the closure of $M$, and therefore, $M$ is a Kakutani-Stone space.

3.5 Corollary. Let $M$ be a closed vector subspace of $C V_{\infty}(X ; \mathbf{R})$. Then, $M$ is a KS.space if, and only if, $M$ is a vector sublattice.

3.6 Proposition. A vector subspace $M$ of $C V_{\infty}(X ; R)$ is a G.space if, and only if, $\bar{M}$ is determined by its restrictions to the two-point subsets of $X$.

Proof. For any vector subspace $M$ of $C V_{\infty}(X ; \mathbf{R})$, it follows from the definition of $G(M)$ that $G(M)$ is determined by its restrictions to the two-point subsets of $X$. Conversely, if $\bar{M}$ is determined by its restrictions to the two-point subsets of $X$, then an obvious modification of the argument presented in the proof of Theorem 3.4 proves that $M$ is a $G$-space.

3.7 Remark. For any vector subspace $M$ of $C V_{\infty}(X ; \mathbf{R})$, it follows from the definition of $G(M)$ that $G(M)$ contains the function sup $(0, f, b)+\inf (0, f, b)$, whenever it contains $f$ and $b$. 
3.8 Theorem. Let $M$ be a vector subspace of $C V_{\infty}(X ; R)$ sucb that $M$ contains sup $(0, f, b)+\inf (0, f, b)$ whenever it contains $f$ and $b$. Then $M$ is a Grothendieck space.

Proof. This result can be proved by modifying conveniently the argument of Lindenstrauss and Wulbert presented in the proof of Theorem $2^{\prime}$ of [5], making use of the fact that, for each $f \in C V_{\infty}(X ; \mathbf{R}), v \in V$, and $\epsilon>0$, the set $K=$ $\{x \in X ; v(x)|f(x)| \geq \epsilon\}$ is compact.

3.9 Remark. It follows from the above results that, if $M$ is an algebra contained in $C V_{\infty}(X ; \mathbf{R})$ such that its closure is a lattice, then $M$ is localizable. More generally, if the closure of $M$ is a $G$-space, then $M$ is localizable.

3.10 Definition. A vector subspace $L \subset C V_{\infty}(X ; E)$ is called a latticial subspace (respectively a Lindenstrauss-Wulbert subspace) if it satisfies the following conditions:

(a) For each $u^{*} \in E^{*}, u \in E$, and $b \in L$, then $u^{*}(b) u \in L$.

(b) There exists a continuous mapping $T: E \times E \rightarrow E$ such that for some $v \in E, T(v, v) \neq 0$ and, for all pairs $a, b \in \mathbf{R}, T(a v, b v)=\max (a, b) T(v, v)$ (respectively $T(a v, b v)=(\max (0, a, b)+\min (0, a, b)) T(v, v))$ and $T(f, b) \in L$, for all $f, b \in L$.

3.11 Proposition. Let $L$ be a (closed) vector subspace of $C V_{\infty}(X ; E)$ and let $M=\left\{u^{*}(f) ; u^{*} \in E^{*}, f \in L\right\}$. Consider the following statements.

(1) $L$ is a latticial subspace.

(2) $M$ is a (closed) KS-space sucb that $M \otimes E \subset L$.

(3) $L$ is a Lindenstrauss.Wulbert subspace.

(4) $M$ is a (closed) G-space such that $M \otimes E \subset L$.

Then, (1) is equivalent to (2), and (3) is equivalent to (4).

Proof. The proofs are similar to the proof of Lemma 2.2, and make use of Theorems 3.4 and 3.8.

3.12 Proposition. Let $L$ be a vector subspace of $C V_{\infty}(X ; E)$. Then $K S(L)$ is a latticial subspace, and $G(L)$ is a Lindenstrauss.Wulbert subspace.

Proof. Let $u^{*} \in E^{*}, u \in E$, and $f \in K S(L)$ be given. Let $b=u^{*}(f) u_{0}$ For any pair $(x, y) \in K S_{L}$ we have: $b(x)=u^{*}(f(x)) u=g(x, y) u^{*}(f(y)) u=g(x, y) b(y)$. Hence $b \in K S(L)$. Now choose $v^{*} \in E^{*}$ and $v \in E$ such that $v^{*}(v)=1$. Define $T: E \times E \rightarrow E$ by setting $T(s, t)=\max \left(v^{*}(s), v^{*}(t)\right) v$ for all $s, t \in E$. Then $T$ is continuous and $T(v, v)=v \neq 0$, and $T(a v, b v)=\max (a, b) T(v, v)$. Let now f, $b \in K S(L)$. It follows that $T(f(x), b(x))=\max \left(v^{*}(f(x)), v^{*}(b(x))\right) v=$ $\max \left(g(x, y) v^{*}(f(y)), g(x, y) v^{*}(b(y))\right) v=g(x, y) T(f(y), b(y))$, for all $(x, y) \in K S_{L}$, so $T(f, b)$ belongs to $K S(L)$. Therefore $K S(L)$ satisfies (a) and (b) of Definition 3.10. The proof that $G(L)$ is a Lindenstrauss-Wulbert subspace is similar. 
3.13 Corollary. Let $L$ be a vector subspace of $C V_{\infty}(X ; E)$. If $L$ is a $K S$-space (respectively a G-space), then its closure is a latticial subspace (respectively a Lindenstrauss.Wulbert subspace).

3.14 Theorem. Let $L$ be a vector subspace of $C V_{\infty}(X ; E)$, wbicb is a latticial subspace (respectively a Lindenstrauss.Wulbert subspace). Assume that $A(K S)$ (respectively $A(G)$ ) separates the equivalence classes modulo $K S_{L}$ (respectively $G_{L}$ ). Then $L$ is a Kakutani-Stone space (respectively a Grotbendieck space).

Proof. Let $\Delta \in\{K S, G\}$. Let $M=\left\{u^{*}(f) ; u^{*} \in E^{*}, f \in L\right\}$. From Proposition $3.11, M$ is a $\Delta$-space contained in $C V_{\infty}(X ; \mathbf{R})$ such that $M \otimes E C L$. Since $\Delta(M)=\bar{M}, \bar{M} \otimes E$ is an $A(\Delta)$-module. Let $f \in C V_{\infty}(X ; E)$ belong to $\Delta(L)$. Let $Y \subset X$ be an equivalence class modulo $X / A(\Delta)$. Since $A(\Delta)$ separates the equivalence classes modulo $\Delta_{L}$, the set $Y$ is contained in some equivalence class modulo $\Delta_{L}$. Fix $x_{0} \in Y$. If $f\left(x_{0}\right)=0$, then $f(x)=0$ for all $x \in Y$, and the function $0 \in M \otimes E$ agrees with $f$ throughout $Y$. If $f\left(x_{0}\right) \neq 0$, there is some $b \in L$ such that $b\left(x_{0}\right) \neq 0$. Choose $u^{*} \in E^{*}$ such that $u^{*}\left(b\left(x_{0}\right)\right)=1$. Let $k=u^{*}(b) f\left(x_{0}\right)$ $\epsilon M \otimes E$. Then $k$ agrees with $/$ throughout $Y$. By Lemma $2.8, f$ belongs to the closure of $\bar{M} \otimes E$. Since $\bar{M} \otimes E$ is contained in the closure of $L, L$ is a $\Delta$-space

3.15 Remark. The hypothesis that $A(\Delta)$ separates the equivalence classes modulo $\Delta_{L}$ is equivalent to the hypothesis that $C_{b}(F ; \mathbf{R})$ separates points, where $F$ is the quotient space of $X$ by the equivalence relation $\Delta_{L}$. One instance in which this occurs is the following. Suppose that our completely regular Hausdorff space $X$ is in fact a normal space and that $\Delta_{L}$ is an upper semicontinuous relation, i.e., $\Delta_{L}$ is closed (Willard [12, Theorem 9.9]). Then, $F$ is a normal Hausdorff space too (Engelking [4, Theorem 5, p. 85]). Another instance occurs when $\Delta_{L}$ reduces to the diagonal. Indeed, in this case, $A(\Delta)$ is $C_{b}(X ; \mathbf{R})$. Notice that, in this case, as was pointed out in 2.10, $\Delta(L)$ is the set of all $f \in C V_{\infty}(X ; E)$ which vanish on the set $Z(L)=\{x \in X ; b(x)=0$ for all $b \in L\}$.

3.16 Corollary. Let $L$ be a vector subspace of $C V_{\infty}(X ; E)$, whicb is a latticial subspace (respectively a Lindenstrauss-Wulbert subspace). Suppose that $K S_{L}$ (respectively $G_{L}$ ) reduces to the diagonal. Then, $f \in C V_{\infty}(X ; E)$ belongs to the closure of $L$, if and only if, $f$ vanishes on the set $Z(L)=$ $\{x \in X ; b(x)=0$ for all $b \in L\}$.

3.17 Corollary. Let $L$ be as in Corollary 3.16, and suppose that $K S_{L}$ (respectively $G_{L}$ ) reduces to the diagonal and that $L$ is everywbere different from zero. Then $L$ is dense in $C V_{\infty}(X ; E)$.

3.18 Remark. Our Theorem 3.14 does not contain Theorem 1.13 of Blatter 
[2]. The argument presented there rests on the fact that $C_{\infty}(X ; \mathbf{R})$ and the $K S$-hull (respectively the $G$-hull) of $M$ have the approximation property. However, his arguments can be used to prove the following generalization of Theorem 1.13 of [2].

3.19 Theorem. Let $X$ be a completely regular Hausdorff k-space, and let $E$ be a complete locally convex Hausdorff space. Suppose that for every compact subset $K \subset X$ and every $t>0$, there exists $v \in V$ such that $v(x) \geq t$ for all $x \in K$. Let $L$ be a vector subspace of $C V_{\infty}(X ; E)$ which is a latticial subspace, respectively a Lindenstrauss.Wulbert subspace. Then $L$ is a KS.space, respectively a G-space.

Proof. The space $C V_{\infty}(X ; \mathbf{R})$ has the approximation property, by Theorem 3 , \$, Bierstedt [1].

4. Examples and applications. Let $V$ be the set of characteristic functions of compact subsets of $X$. The Nachbin space $C V_{\infty}(X ; E)$ is just $C(X ; E)$ endowed with the compact-open topology. A subset $L C C(X ; E)$ is called selfadjoint if $M=\left\{u^{*}(f) ; u^{*} \in E^{*}, f \in L\right\}$ is a selfadjoint subset of $C(X ; \mathbf{K})$.

4.1 Theorem (Weierstrass-Stone). Let $L$ be a selfadjoint polynomial subalgebra of $C(X ; E)$. A function $f \in C(X ; E)$ belongs to the closure of $L$ if, and only if, the following conditions bold:

(1) For every $x, y \in X$ sucb that $f(x) \neq f(y)$, there is $b \in L$ sucb that $b(x) \neq b(y)$.

(2) For every $x \in X$, such that $f(x) \neq 0$, there is $b \in L$ sucb that $b(x) \neq 0$.

Proof. Since every selfadjoint subalgebra of $C(X ; K)$ is localizable $[6$, Theorem $1, \$ 30]$, Theorem 2.9 implies that $L$ is a Weierstrass-Stone space, and therefore its closure is precisely WS $(L)$. It remains only to notice that $f \in W S(L)$ if, and only if, conditions (1) and (2) are satisfied.

4.2 Corollary. Let $L$ be a selfadjoint polynomial subalgebra of $C(X ; E)$, separating and everywbere different from zero on $X$. Then $L$ is dense in $C(X ; E)$.

4.3 Corollary. Let $A$ be a selfadjoint subalgebra of $C(X ; K)$, separating and everywbere different from zero on $X$. Then $A \otimes E$ is dense in $C(X ; E)$. In particular, $C(X ; \mathrm{K}) \otimes E$ is dense in $C(X ; E)$.

Suppose that $X$ is the Cartesian product of two completely regular Hausdorff spaces $X_{1}$ and $X_{2}$. Let $L$ be the set of all finite sums of functions of the form $\left(x_{1}, x_{2}\right) \mapsto f\left(x_{1}\right) b\left(x_{2}\right)$, where $f \in C\left(X_{1} ; K\right)$ and $b \in C\left(X_{2} ; E\right)$. Dieudonné's approximation theorem on Cartesian products, stating that $C\left(X_{1} ; K\right) \otimes C\left(X_{2} ; E\right)$ is dense in $C\left(X_{1} \times X_{2} ; E\right)$, is now an easy consequence of Corollary 4.2. 
4.4 Theorem. Let $E$ and $F$ be two locally convex Hausdorff spaces over the reals. The closure in the compact-open topology of the set of all continuous poly. nomials $p$ of finite type from $E$ into $F$, with $p(0)=0$, consists of all mappings $f \in C(E ; F)$ sucb that $f(0)=0$.

Proof. This follows from a direct application of Theorem 4.1.

4.5 Theorem (Infinite-dimensional Weierstrass polynomial approximation theorem). Let $E$ and $F$ be two locally convex Hausdorff spaces over the reals. Then $P f(E ; F)$ is dense in $C(E ; F)$.

Proof. Apply Corollary 4.2 to $L=P(E ; F)$.

4.6 Remark. The space $P_{f}(E ; F)$ is not selfadjoint in the complex case. Instead of $P_{f}(E ; F)$ one has to consider the vector subspace $P_{f}^{*}(E ; F)$ defined as follows. For each integer $n \geq 1, P_{f}^{*}\left({ }^{n} E ; F\right)$ denotes the vector subspace of $C(E ; F)$ generated by the set of all maps of the form $x \mapsto u^{*}(x)^{n} u$, where $u \in F$ and $u^{*}$ or its complex conjugate belongs to $E^{*}$. Then $P_{f}^{*}(E ; F)$ is, by definition, the vector subspace generated by the union of all $P_{f}^{*}\left({ }^{n} E ; F\right)$ with $n \geq 1$ and the constant maps. Theorem 4.5 now holds with $P_{f}^{*}(E ; F)$ substituted for $P_{f}(E ; F)$.

Let $X$ be a locally compact Hausdorff space and let $V=C_{\infty}^{+}(X)$, the set of all positive continuous functions vanishing at infinity on $X$. The Nachbin space $C V_{\infty}(X ; E)$ is then $C_{b}(X ; E)$ endowed with the strict topology $\beta$. Again we are in the bounded case of the approximation problem, and therefore every selfadjoint subalgebra of $C_{b}(X ; K)$ is localizable under itself in the strict topology. Hence Theorem 4.1 and its corollaries hold when $C_{b}(X ; E)$ with the strict topology is substituted for $C(X ; E)$ with the compact-open topology.

5. Extension theorems. Let $Y$ be a closed subset of $X$. Then, for every weight $v$ on $X$, its restriction to $Y$ is a weight on $Y$. If $V$ is a set of weights on $X$, we denote by $V \mid Y$ the set of weights on $Y$ that are restriction to $Y$ of some element of $V$. The corresponding Nachbin space $C(V \mid Y)_{\infty}(Y ; E)$ will be denoted by $C V_{\infty}(Y ; E)$. Let $L$ be a vector subspace of $C V_{\infty}(X ; E)$. A function $f \epsilon$ $C V_{\infty}(Y ; E)$ is said to be extensible in $L$ if there exists $b \in L$ such that $b \mid Y=f_{0}$ i.e. $b(x)=f(x)$ for all $x \in Y$. The set of all such extensible functions is denoted by $L \mid Y$. Notice that, if $L$ is a polynomial algebra, or a latticial subspace, or a Linderstrauss-Wulbert subspace, the same is true of $L \mid Y$ as subspace of $C V_{\infty}(Y ; E)$.

5.1 Theorem. Let $Y$ be a closed subset of a completely regular Hausdorff space $X$. Let $L$ be a vector subspace of $C V_{\infty}(X ; E)$ which is a polynomial algebra such that every $b \in L$ is bounded on the support of every $v \in V$. Assume that $L$ is selfadjoint and that $L \mid Y$ is closed in $C V_{\infty}(Y ; E)$. Then $f \in C V_{\infty}(Y ; E)$ is extensible in $L$ if, and only if, the following conditions are satisfied: 
(1) For any $x, y \in Y$ sucb that $f(x) \neq f(y)$, there is $b \in L$ such that $b(x)$ $\neq b(y)$.

(2) For any $x \in Y$ sucb that $f(x) \neq 0$, there is $b \in L$ sucb that $b(x) \neq 0$.

Proof. Under the hypothesis made, $L \mid Y$ is a WS-space.

5.2 Corollary. Let $Y$ and $L$ be as in Theorem 5.1. If $L \mid Y$ is separating and everywbere different from zero on $Y$, then every $f \in C V_{\infty}(Y ; E)$ is extensible in $L$.

5.3 Theorem. Let $Y$ be a compact subset of a completely regular Hausdorff space $X$, let $E$ be a Banach space, and let $f \in C(Y ; E)$. There is $b \in C_{b}(X ; E)$ such that $b \mid Y=f$ and $\|f\|_{Y}=\|b\|_{X}$.

Proof. Let $L=C_{b}(X ; E) \subset C(X ; E)$. An obvious modification of the argument given in Stone $[11, \mathrm{p} .64]$ proves that $L \mid Y$ is closed in $C(Y ; E)$. Since $X$ is completely regular, $C_{b}(X ; E)$ is separating and everywhere different from zero on $X$, in particular on $Y$. By Corollary 5.2 , every $f \in C(Y ; E)$ is extensible in $C_{b}(X ; E)$. The final statement follows from the fact that for any function $f \epsilon$ $C(X ; E)$, which is bounded on $Y$, there is $b \in C_{b}(X ; E)$ such that $b \mid Y=f$ and $\|f\|_{Y}=\|b\|_{X}$.

5.4 Theorem. Let $Y$ be a closed subset of a normal Hausdorff space $X$, let $E$ be a Danach space, and let $f \in C(Y ; E)$ be a compact mapping. There exists a compact mapping $b \in C(X ; E)$ sucb that $b \mid Y=f$ and $b(X)$ is contained in the closed convex bull of $f(Y)$.

Proof. If $\beta X$ and $\beta Y$ denote the Stone-Čech compactifications of $X$ and $Y$ respectively, it is known that $\beta Y$ identifies with the closure of $Y$ in $\beta X$, (see [10]) and that a mapping $f \in C(Y ; E)$ has an extension to $\beta Y$ if, and only if, $f(Y)$ is precompact. Hence we can extend $f$ to $\beta Y$ though a function $w \in C(\beta Y ; E)$. By Theorem 5.3 we can extend $w$ to $\beta X$. If $v$ denotes such an extension, let $u=v \mid X$. Then $u \in C(X ; E)$ and $u \mid Y=f$. Since $E$ is a Banach space, the closed convex hull $K$ of $f(Y)$ is compact and, therefore, a retract of $E$ (see [3]). Let $r$ be a retraction of $E$ onto $K$. Then $b=r(u)$ is a compact mapping that answers all the requirements.

5.5 Theorem. Let $Y$ be a closed subset of a completely regular Hausdorff space $X$, let $E$ be a Banacb space. A function $f \in C_{\infty}(Y ; E)$ is extensible in $C_{\infty}(X ; E)$ if, and only if, the following conditions bold:

(1) For any $x, y \in Y$ such that $f(x) \neq f(y)$, there is $b \in C_{\infty}(X ; E)$ sucb that $b(x) \neq b(y)$.

(2) For any $x \in Y$ sucb that $f(x) \neq 0$, there is $b \in C_{\infty}(X ; E)$ sucb that $b(x) \neq 0$. 
If $f \in C_{\infty}(Y ; E)$ is extensible, we can choose an extension $b \in C_{\infty}(X ; E)$ sucb that $\|f\|_{Y}=\|b\|_{X}$.

Proof. Take $L=C_{\infty}(X ; E)$. As in Theorem 5.3, $L \mid Y$ is closed in $C_{\infty}(Y ; E)$. Theorem 5.5 follows then from Theorem 5.1.

5.6 Corollary. Let $Y$ be a closed subset of a locally compact Hausdorff space $X$, let $E$ be a Banach space. Then, every $f \in C_{\infty}(X ; E)$ is extensible in $C_{\infty}(X ; E)$, and we can choose an extension with the same norm.

Proof. Since $X$ is locally compact, the space $C_{\infty}(X ; E)$ is separating and everywhere different from zero.

\section{BIBLIOGRAPHY}

1. K.-D. Bierstedt, Gewi chtete Räume Stetiger Vektorwertiger Funktionen und das Injektive Tensorprodukt, Ph. D. Dissertation, Johannes Gutenberg.Universität in Mainz, 1970.

2. J. Blatter, Grothendieck spaces in approximation theory, Mem. Amer. Math. Soc. No. 120 (1972).

3. J. Dugundji, An exten sion of Tietze's theorem, Pacific J. Math. 1 (1951), 353-367. MR 13, 373.

4 R. Engelking, Outline of general topology, PWN, Warsaw, 1965; English transl., North-Holland, Amsterdam; Interscience, New York, 1968. MR 36 \#4508; 37 \#5836.

5. J. Lindenstrauss and D. E. Wulbert, $O_{n}$ the classification of the $B$ anach spaces whose duals are $L_{1}$ spaces, J. Functional Analysis 4 (1969), 332-349. MR 40 \#3274.

6. L. Nachbin, Elements of approximation theory, Van Nostrand Math. Studies, no. 14, Van Nostrand, Princeton, N. J., 1967. MR 36 \#572.

7. Weighted approximation for function algebras and quasi-analytic mappings, Function Algebras (Proc. Internat. Sympos. on Function Algebras, Tulane Univ., 1965), Scott-Foresman, Chicago, Ill., 1966, pp. 330-333. MR 33 \#7835.

8. L. Nachbin, J. B. Prolla and S. Machado, Conceming weighted approximation, vector fibrations, and algebras of operators, J. Approximation Theory 6 (1972), 80-89.

9. A. Pelczyński, A gen eralization of Stone's theorem on approximation, Bull. Acad. Polon. Sci. C. III S(1957), 105-107. MR 19, 135.

10. M. H. Stone, On the compactification of topological spaces, Ann. Soc. Polon. Math. 21 (1948), 153-160. MR 10, 137.

11. - The gen eralized Wei erstrass approximation theorem. In Buck, R. C., Studies in Modern Analysis, MAA Studies in Mathematics 1 (1962), 30-87.

12. S. Willard, General topology, Addi son-Wesley, Reading, Mass., 1970. MR 41 \#173,

INSTITUTO DE MATEMATICA, UNIVERSIDADE FEDERAL DO RIO DE JANEIRO, CAIXA POSTAL 1835, ZC-00, 20000 RIO DE JANEIRO, GB, BRASIL. 